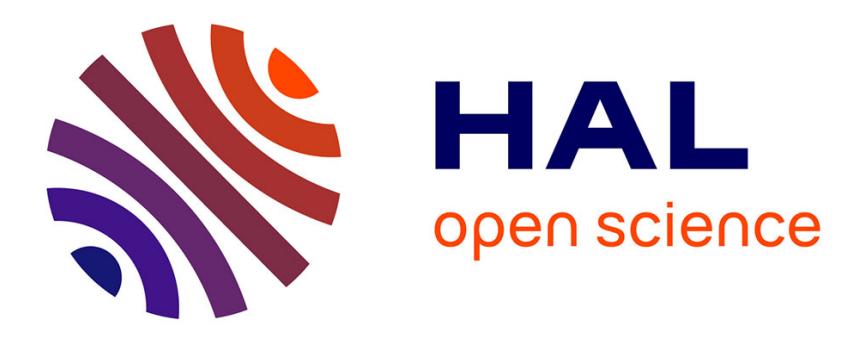

\title{
Estimation de l'action du charançon des siliques (Ceuthorrhynchus assimilis Payk.) sur la productivité du colza d'hiver I. Aspects méthodologiques
}

\author{
Jacques Lerin, Eric Rivault
}

\section{To cite this version:}

Jacques Lerin, Eric Rivault. Estimation de l'action du charançon des siliques (Ceuthorrhynchus assimilis Payk.) sur la productivité du colza d'hiver I. Aspects méthodologiques. Agronomie, 1982, 2 (10), pp.1005-1014. hal-00884342

\section{HAL Id: hal-00884342 \\ https://hal.science/hal-00884342}

Submitted on 1 Jan 1982

HAL is a multi-disciplinary open access archive for the deposit and dissemination of scientific research documents, whether they are published or not. The documents may come from teaching and research institutions in France or abroad, or from public or private research centers.
L'archive ouverte pluridisciplinaire HAL, est destinée au dépôt et à la diffusion de documents scientifiques de niveau recherche, publiés ou non, émanant des établissements d'enseignement et de recherche français ou étrangers, des laboratoires publics ou privés. 


\title{
Estimation de l'action du charançon des siliques (Ceuthorrhynchus assimilis Payk.) sur la productivité du colza d'hiver I. Aspects méthodologiques
}

\author{
Jacques LERIN \\ avec la collaboration technique d'Eric RIVAULT \\ I.N.R.A., Laboratoire de Zoologie, F. 86600 Lusignan
}

\section{RÉSUMÉ \\ Etude méthodologique, Brassica napus $L$. var. oleifera Metzger, \\ Ceuthorrhynchus assimilis Payk, \\ Pollinisation entomophile, Composantes du rendement, Essai sous cage, \\ Distribution spatiale, \\ Rapports plante insecte.}

Cet article présente quelques problèmes méthodologiques relatifs à la détermination des pertes de rendement du colza dues au charançon des siliques dans des expériences en cage. La pollinisation doit être assurée par l'adjonction d'insectes pollinisateurs malgré le caractère autofertile du colza.

Les variations du nombre de siliques et du nombre de graines par silique masquent l'action du charançon. Afin de pouvoir analyser les résultats dans de bonnes conditions d'application des tests statistiques, la transformation en logarithmes du nombre de siliques et du rendement et en racine carrée du nombre de larves est nécessaire pour stabiliser les variances.

La répartition des larves sur les plantes et l'effet du développement de la plante sur l'abondance de la descendance des femelles ont été particulièrement étudiés. Une méthode d'échantillonnage est préconisée pour déterminer le niveau d'infestation de lots de plantes.

\section{SUMMARY}

Methodological study, Brassica napus $L$. var. oleifera Metzger,

Ceuthorrhynchus assimilis Payk,

Insect pollination,

Yield components,

Cage experiment,

Spatial distribution,

Insect plant relationship.
Assessment of yield loss in winter rape due to seed-pod weevil (Ceuthorrhynchus assimilis Payk.) I. - Methodological aspects

This paper presents some methodological problems in the determination of yield losses in rape caused by the seed-pod weevil in a cage experiment. Although rape is self-fertile, bumble-bees had to be introduced to improve pollination and reduce the variance of the number of pods per plant.

Variation in the number of pods and in the number of seeds per pod tended to conceal the damage caused by the seed-pod weevil. High numbers of plants had to be assessed to show the impact of the secd-pod weevil on yield. Logarithmic transformation of the number of pods and yield per plant and square-root transformation of the number of larvae per plant were necessary to avoid links between means and variances.

The distribution of larvae on the plants is described and a sampling method is proposed to determine the level of attack of a plant lot.

\section{INTRODUCTION}

Le travail méthodologique entrepris depuis 1978 a permis de cerner un certain nombre de problèmes relatifs à l'étude des pertes de rendement du colza, Brassica napus $\mathrm{L}$. var. oleifera Metzger, dues au charançon des siliques, Ceuthorrhynchus assimilis Payk., et d'y apporter des solutions.

Le but est l'estimation des pertes de rendement par plante causées par les larves et non la détermination des dégâts larvaires par silique, résultat acquis, puisqu'on sait qu'une larve détruit entre 3,5 et 5 graines durant son développement (DMOCH, 1965 ; LACOTTE, 1973). L'une des caractéristiques du colza est son extrême plasticité. C'est une plante qui possède un grand pouvoir de récupération après un stress, du moins pour la variété « Jet neuf » actuellement la plus cultivée en France.

A la sortie de l'hiver, seulement 60 p. 100 de la variance du rendement en graine peuvent être prévus à partir des caractéristiques végétatives de la plante (BUSON, 1979). On peut prédire, grossièrement, que les plantes à petit diamètre au collet $(<10 \mathrm{~mm})$ dont le nombre de feuilles est faible, auront en général une production médiocre. En revanche, le rendement des plantes à développement moyen ou grand dépendra fortement des conditions climatiques ultérieures notamment des périodes de sécheresse depuis l'apparition des boutons floraux jusqu'à la maturation des siliques 
(Dembinski, 1970 ; Rollier, 1974 ; Clarke \& SimPSON, 1978).

Durant la floraison, la plante produit beaucoup plus de boutons, de fleurs et de siliques qu'elle ne pourra en faire évoluer pleinement par la suite. Il se produit durant cette période un certain nombre d'avortements d'organes d'autant plus important que les inflorescences sont plus tardives. Jusqu'à 50 p. 100 des organes fructifères avortent avant la récolte (LACOTTE, 1973). Cependant, lorsqu'une attaque d'insectes se produit ou que des conditions climatiques défavorables interviennent (grêle, gel pendant la floraison), détruisant des organes fructifères jeunes, les avortements "physiologiques» diminuent. L'explication habituelle de ce phénomène est l'équilibre entre la demande croissante en métabolites par les siliques et la fourniture de ceux-ci par les parties végétatives de la plante.

RISBEC (1952) notait : "Une étude de l'action des insectes devra donc être dominée par la connaissance des conditions de la plante et il faudra garder à l'esprit la notion d'une limite dans le temps... avant laquelle tout dégât infligé au colza pourra être réparé dans certaines conditions et après laquelle tout dégât sera définitif ». DMOCH (1965) confirmait cette appréciation en observant que les adultes n'occasionnent que très rarement des dégâts d'importance économique: en effet, la phase intense d'alimentation nécessaire à la maturation sexuelle intervient généralement pendant la floraison lorsque le colza a encore tout son potentiel de récupération. WILLIAMS \& FREE (1979) ont ainsi montré qu'une perte de 60 p. 100 des siliques jeunes (par abscission manuelle) n'a pas d'influence sur le rendement final, si la plante est placée dans des conditions telles qu'elle puisse compenser cette perte.

Tout se passe comme si la plante produisait des boutons jusqu'à ce qu'un niveau minimal de production soit atteint. Ce niveau dépend de la vigueur de la plante. L'élaboration du rendement pourrait donc être modifié par les ravageurs. Comme, de plus, la durée de floraison semble avoir une influence sur l'abondance des pontes du charançon des siliques (RISBEC, 1952 ; BONNEMAISON, 1957 ; DMOCH, $1965)$, on est en présence d'interactions complexes entre plante et insectes.

\section{MATÉRIEL ET MÉTHODE}

\section{A. Cages}

Les expériences ont lieu en cages de $8 \mathrm{~m}^{3}(2 \times 2 \times 2 \mathrm{~m})$, recouvertes de grillage à mailles fines $(14 \mathrm{au} \mathrm{cm})$, qui modifient probablement moins le comportement des insectes qu'un manchon en mousseline enserrant une plante isolée. La cage grillagée est pour l'instant le seul moyen permettant d'isoler les plantes des attaques d'autres insectes tout en maintenant en place une densité contrôlée du ravageur étudié et en modifiant le moins possible les paramètres climatiques, notamment la température et l'humidité. L'inconvénient majeur est la quasi-disparition du vent et de l'agitation des plantes.

\section{B. Plantes}

La variété de colza «Jet neuf » est utilisée pour l'expérimentation. En 1979, des plantes au stade rosette, provenant d'un champ, ont été repiquées à raison de 2 par caissette. Chaque caissette mesure $30 \times 10 \times 15 \mathrm{~cm}$ et contient un mélange constitué de $1 / 3$ de terre franche, $1 / 3$ de sable et $1 / 3$ de terreau. En 1980, les graines ont été semées directement dans les caissettes en 2 poquets. Après la levée, 2 plantes ont été conservées. Pour ces 2 années, 8 caissettes ont été disposées dans les cages (suivant le même schéma et après tirage au sort); on a donc 16 plantes par cage au départ. Lors de la récolte, notamment en 1980, un certain nombre de plantes ont du être éliminées des résultats (12/192) en raison de l'absence d'inflorescence principale, phénomène dû à une maladie cryptogamique. La mise en culture du mycelium présent sur les pivots nécrosés des plantes au stade rosette a permis de montrer qu'il s'agissait de Sclerotinia sp.

\section{Insectes}

Les insectes sont prélevés dans la nature dès que l'on obtient les premières captures en piège jaune. Ils sont maintenus sur des inflorescences de colza ne présentant que des boutons ou des fleurs. Les insectes qui « s'accouplent » sont retirés et groupés en lots qui correspondent aux densités d'adultes par plante qui seront utilisées dans chaque cage. L'hétérosexualité des " couples » a été contrôlée par dissection d'échantillons : chaque année le résultat a été de $100 \mathrm{p} .100$. Cette procédure est rendue nécessaire par l'absence de caractère simple permettant de distinguer les mâles des femelles. Les caractéristiques des infestations ont été regroupées dans le tableau 1. En 1980, les densités d'adultes ont été choisies de manière à « encadrer » le seuil d'intervention (1 charançon par plante) préconisé par le CETIOM (Centre technique interprofessionnel des Oléagineux métropolitains). Le traitement que l'on fait subir à chaque cage est tiré au hasard.

TABLEAU 1

Caractéristiques des infestations.

Characteristics of the infestations.

\begin{tabular}{ccccc}
\hline \hline Année & $\begin{array}{c}\text { Nombre } \\
\text { de } \\
\text { traitements }\end{array}$ & $\begin{array}{c}\text { Nombre de } \\
\text { cages par } \\
\text { traitements }\end{array}$ & $\begin{array}{c}\text { Nombre de charançons } \\
\text { introduits par } \\
\text { traitements }\left(^{*}\right)\end{array}$ & Stade phénologique \\
\hline 1979 & 2 & 2 & $0-40$ & 10 premières siliques \\
bosselées (G4) \\
boutons jaunes (E) \\
boutons jaunes (E)
\end{tabular}

(*) Sex ratio $=1$ 
TABLEAU 2

Moyennes et variances des variables principales mesurées sur les plantes sans charançons. Means and variances of the main parameters measured on plants without seed-pod weevils.

\begin{tabular}{|c|c|c|c|c|c|c|c|c|c|}
\hline & & \multicolumn{2}{|c|}{ ST } & \multicolumn{2}{|c|}{$\mathrm{G} / \mathrm{ST}$} & \multicolumn{2}{|c|}{ PT } & \multicolumn{2}{|c|}{ NI } \\
\hline & & $X$ & $\mathbf{X}^{\prime}$ & $\mathbf{X}$ & $\mathbf{X}^{\prime}$ & $X$ & $\mathbf{X}^{\prime}$ & $\mathrm{X}$ & $\mathbf{X}^{\prime}$ \\
\hline $\begin{array}{l}1979 \\
\mathrm{n}=30\end{array}$ & moy & $\begin{array}{l}111,53 \\
8258\end{array}$ & $\begin{array}{l}4,6991 \\
0,0675\end{array}$ & $\begin{array}{r}11,96 \\
3,75\end{array}$ & $\begin{array}{l}2,4697 \\
0,0260\end{array}$ & 6,82 & $\begin{array}{l}1,9031 \\
00788\end{array}$ & 4,10 & 1,3522 \\
\hline $\begin{array}{l}1980 \\
\text { pollinisé } \\
n=31\end{array}$ & $\begin{array}{l}\text { moy } \\
\text { var }\end{array}$ & $\begin{array}{r}285,0 \\
5102,0\end{array}$ & $\begin{array}{l}5,6225 \\
0,0628\end{array}$ & $\begin{array}{l}16,94 \\
11,57\end{array}$ & $\begin{array}{l}2,8096 \\
0,0422\end{array}$ & $\begin{array}{l}29,19 \\
78,80\end{array}$ & $\begin{array}{l}3,3276 \\
0,0991\end{array}$ & $\begin{array}{l}8,90 \\
1,690\end{array}$ & $\begin{array}{l}2,1760 \\
0,0217\end{array}$ \\
\hline $\begin{array}{l}1980 \\
\text { non pollinisé } \\
n=29\end{array}$ & $\begin{array}{l}\text { moy } \\
\text { var }\end{array}$ & $\begin{array}{r}381,9 \\
45598,7\end{array}$ & $\begin{array}{l}5,8183 \\
0,2492\end{array}$ & $\begin{array}{r}10,64 \\
5,96\end{array}$ & $\begin{array}{l}2,3350 \\
0,0668\end{array}$ & $\begin{array}{r}26,01 \\
197,87\end{array}$ & $\begin{array}{l}3,1082 \\
0,3376\end{array}$ & $\begin{array}{c}10,14 \\
4,409\end{array}$ & $\begin{array}{l}2,2960 \\
0,0418\end{array}$ \\
\hline
\end{tabular}

\section{$\mathrm{X}$ données brutes, $\mathrm{X}^{\prime}$ données transformées}

ST nombre total de siliques par plante

G/ST nombre de graines par silique

PT production totale par plante (en $\mathrm{g}$ pour les données brutes)

NI nombre d'inflorescences

\section{Méthode de prélèvement, comptages}

Les inflorescences de chaque plante sont prélevées une à une au fur et à mesure qu'elles arrivent à maturité. On note :

$S$ nombre de siliques.

L nombre de larves estimé par le nombre de trous de sortie relevés sur les siliques.

$P$ poids de graine de l'inflorescence après passage à l'étuve à $60^{\circ} \mathrm{C}$ pendant $48 \mathrm{~h}$.

$\mathrm{G}$ nombre de graines saines (les fragments de graines et les graines perforées ou trop petites sont éliminées).

La notation des inflorescences est la suivante :

IP inflorescence principale.

IS $_{1}$ à IS $\mathbf{S}_{\mathrm{i}}$ inflorescences secondaires par ordre d'apparition sur la plante de haut en bas.

L'indice $\mathrm{T}$ indique qu'il s'agit des données de la plante entière ; par exemple $S_{T}$ est le nombre de siliques total d'une plante.

\section{RÉSULTATS ET DISCUSSION}

\section{A. Pollinisation}

En 1979 , le nombre de graines par silique $(\mathrm{G} / \mathrm{S})$ et le nombre de siliques par plante en cage étaient inférieurs à ceux enregistrés au champ. Lors d'une expérience complémentaire sur colza de printemps, les résultats enregistrés ont montré une nette augmentation de ces 2 données dans les lots infestés par le charançon des siliques. Si l'augmentation du nombre de siliques pouvait apparaître comme une « compensation » de la plante due à la présence du ravageur, celle du nombre de graines par silique devait être due à une meilleure pollinisation. Ce résultat paradoxal pouvait provenir d'un effet pollinisateur des ravageurs qui passent une bonne partie de la journée dans les fleurs : la nuit pour s'abriter, le jour pour s'accoupler et se nourrir.

C'est pourquoi le dispositif de 1980 comportait des lots avec bourdon et des lots sans bourdon. Leur comparaison a fait l'objet d'une précédente publication (LERIN, 1982). Les conclusions sont que, en l'absence de vent et de pollinisateur, les plantes s'autopollinisent mal (tabl. 2). Les résultats sont particulièrement visibles sur les inflorescences princi- pales qui sont bien isolées les unes des autres. Sur les inflorescences secondaires, les différences sont moins importantes, probablement parce que les frottements entre inflorescences dus au vent résiduel permettent une fécondation accrue (LERIN, 1982).

L'analyse du tableau 2 montre que les variances du nombre de graines par silique sont augmentées par la pollinisation entomophile. Cet effet disparaît après transformation logarithmique de la variable (cf. B). En revanche, la pollinisation a un effet favorable sur les variances du nombre de siliques et du nombre d'inflorescences, qu'elle diminue. C'est l'aspect le plus important puisque les variations du nombre de siliques entre plantes déterminent un fort pourcentage de la variance du rendement (tabl. 3). Il faut noter que, pour des colzas cultivés en champ, le coefficient de détermination peut être encore plus élevé à cause de la grande hétérogénéité des plantes (BUSON, 1979 : $\mathrm{R}^{2}=0,852$ en données brutes, $\mathrm{R}^{2}=0,914$ en logarithmes).

\section{TABLEAU 3}

Corrélations entre nombre total de siliques et production dans les lots sans charançons. Variables tranformées en logarithmes.

Correlation between total number of pods and yield in treatments without seed-pod weevils. Logarithmic transformation.

\begin{tabular}{llll}
\hline \hline & Corrélation & $\begin{array}{c}\text { Coefficient } \\
\text { de détermination }\end{array}$ \\
\hline 1979 & $\mathrm{n}=30$ & 0,85 & 0,72 \\
1980 pollinisé & $\mathrm{n}=31$ & 0,78 & 0,62 \\
1980 non pollinisé & $\mathrm{n}=29$ & 0,90 & 0,82 \\
\hline Estimation moyenne & 0,85 & 0,73 \\
\hline \hline
\end{tabular}

Les différentes estimations de la corrélation entre nombre de siliques et poids de graines par plante ne sont significativement différentes, ni sur les données brutes, ni sur les données transformées en logarithmes. Le même type de relation subsiste dans des conditions de pollinisation différentes et le coefficient de corrélation moyen est une bonne estimation de l'intensité de la liaison entre les 2 variables. La pollinisation diminuant la variance du nombre de siliques, on aura intérêt à toujours assurer une bonne 
nouaison par l'adjonction de pollinisateurs dans les cages afin d'homogénéiser les productions.

\section{B. Transformation de variables}

L'hypothèse de normalité des distributions (soit des données élémentaires, soit des résidus dans un modèle) et d'indépendance des variances et des moyennes sont les 2 conditions nécessaires à une bonne application des tests statistiques usuels. Nous les avons donc examinées sur nos données.

\section{1) Données des inflorescences principales}

La figure 1 montre une dissymétric marquée vers les valeurs faibles du nombre de siliques des inflorescences
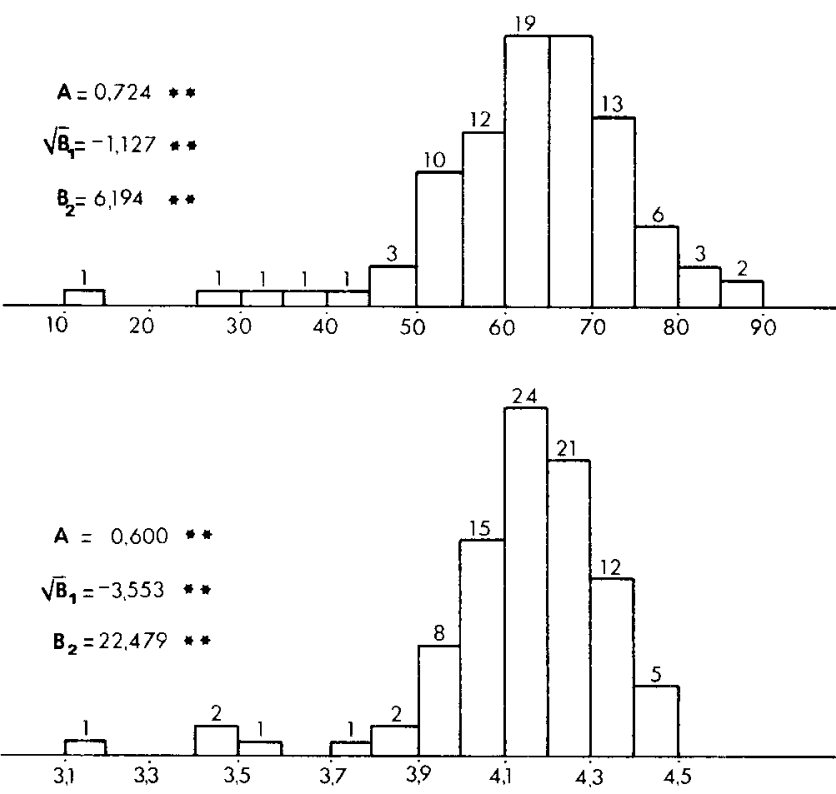

Figure 1

Distribution des nombres de siliques par inflorescence principale sur l'ensemble des plantes pollinisées infestées en 1980, avant (en haut) et après (en bas) transformation en logarithmes.

Distribution of number of pods per main shoot, for all pollinated and attacked plants in 1980, before (above) and after (below) logarithmic transformation.

principales en 1980, qui ne représente que 5 plantes. Ces plantes sont réparties dans tous les traitements, il y a donc peu de chance que leur élimination masque un effet négatif des charançons adultes sur le nombre de siliques. En conséquence, les plantes n'ayant pas 45 siliques (sur l'IP) sont éliminées des calculs ultérieurs. Pour 1979, de la même façon, on considère qu'un nombre de siliques inférieur à 19 est anormal et dû à d'autres causes qu'à une mauvaise pollinisation ou à l'action du charançon : 2 plantes dans les témoins et 2 plantes dans le lot infesté ont été éliminées.

Cette épuration des données est pratiquement obligatoire pour des effectifs aussi faibles: la présence d'une donnée aberrante augmente considérablement la variance du lot. Après élimination de ces données, les variances des différentes cages ne sont plus significativement différentes et la distribution du nombre de siliques sur les inflorescences principales est assimilable à une loi normale : la transformation en logarithme n'est donc pas utile. On aura intérêt à faire les calculs de régression en données brutes, ce qui simplifie l'interprétation des résultats.

\section{2) Données des plantes entières}

Les résultats du tableau 2, concernant le nombre de siliques, montrent que les variances sont très largement supérieures à la moyenne, ce qui indique que les distributions ne suivent pas une loi normale. Il y a, en outre, une liaison forte entre moyenne et variance associées que l'on fait mieux apparaître en mettant en relation les logarithmes de ces paramètres pour chaque cage en 1979 et 1980. Cette relation est la même pour les cages infestées. Dans ces conditions, une transformation logarithmique des données permet de diminuer considérablement les variances et d'assurer leur indépendance vis-à-vis des moyennes (DAGNELIE, 1975). Cette transformation est également nécessaire pour le rendement total.

Les caractéristiques des histogrammes (statistique $\mathrm{A}$ ou $B_{2}$ pour l'aplatissement et $\sqrt{B_{1}}$ pour l'asymétrie) montrent que l'on est plus proche de distributions normales pour le nombre de siliques en 1980 après transformation (fig. 2). En 1979 , les effectifs sont trop faibles pour ce type d'analyse.
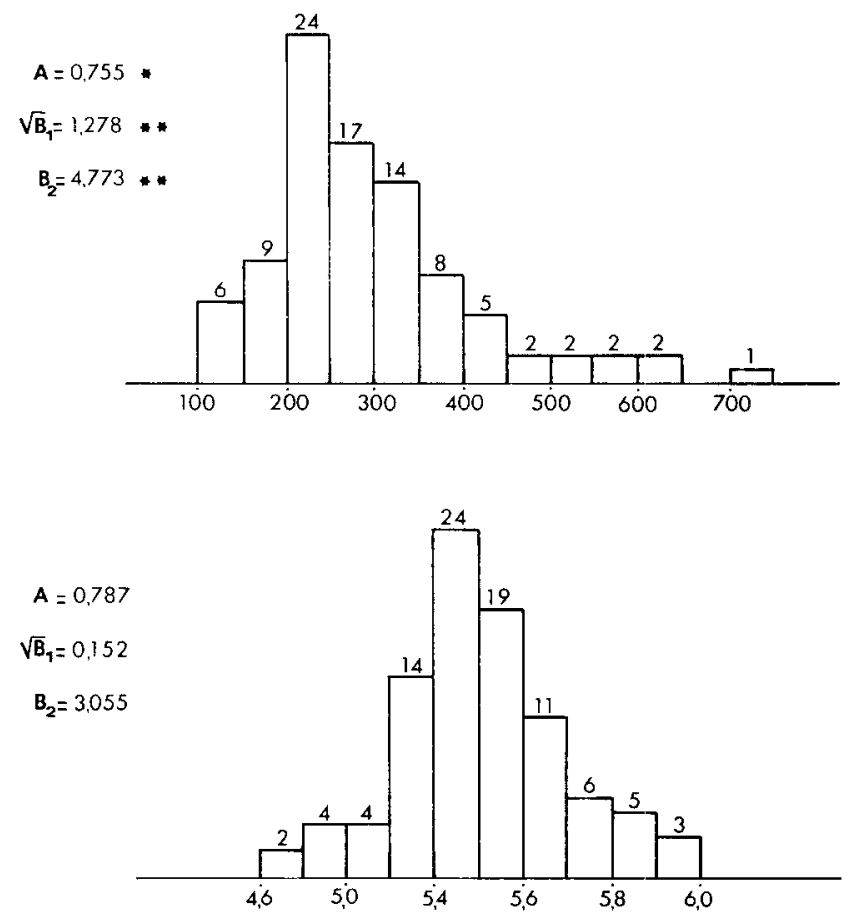

Figure 2

Distribution des nombres de siliques par plante (plantes pollinisées et infestées en 1980, avant et après transformation en logarithmes).

Distribution of number of pods per plant for all pollinated and attacked plants in 1980, before and after logarithmic transformation.

Les différences entre les variances des moyennes des cages ne sont plus statistiquement significatives après transformation (tabl. 4). Pour le nombre de larves par plante, la transformation utilisée est $\sqrt{\mathrm{x}+0,5}$. En effet, si l'on calcule la corrélation entre les logarithmes des variances et des moyennes, on obtient un coefficient de régression $=1$. La loi des puissances de TAYLOR (SOUTHWOOD, 1966; Pielou, 1969 ; Elliot, 1971) montre que le changement de variable assurant une certaine indépendance entre moyennes et variances est :

$$
Z=x^{P} \text { avec } P=1-\frac{\beta}{2},
$$

$Z$ étant la variable transformée, $x$ la variable initiale et $\beta$ le coefficient de régression. La présence de «zéros » dans les 
TABLEAU 4

Test de signification des différences entre variances des cages du lot pollinisé avec ou sans charançons en 1980. Significance test on the differences between variances of cages of the whole batch of pollinated plants.

\begin{tabular}{|c|c|c|c|c|}
\hline \multirow{2}{*}{ Variables } & \multicolumn{2}{|c|}{ Avant transformation } & \multicolumn{2}{|c|}{ Après transformation } \\
\hline & Nbre de siliques & Production & Nbre de siliques & Production \\
\hline $\begin{array}{l}X^{2} \text { de Bartlett } \\
7 \text { d.l. }\end{array}$ & 21,62 & 19,58 & 8,24 & 8,90 \\
\hline Pour 7 d.l. & $X_{0,05}^{2}=14,07$ & $\mathrm{X}_{0,01}^{2}=18,47$ & & \\
\hline
\end{tabular}

dénombrements de larves explique la présence du terme 0,5 .

La symétrie des histogrammes est nettement améliorée par cette transformation (fig. 3 et 4 ). La transformation de $S_{T}$ et $P_{T}$ en logarithmes et de $L_{T}$ en racine carrée nous place dans les meilleures conditions pour les analyses statistiques habituelles (variance, régressions) : distributions proches de la loi normale et variances indépendantes des moyennes.

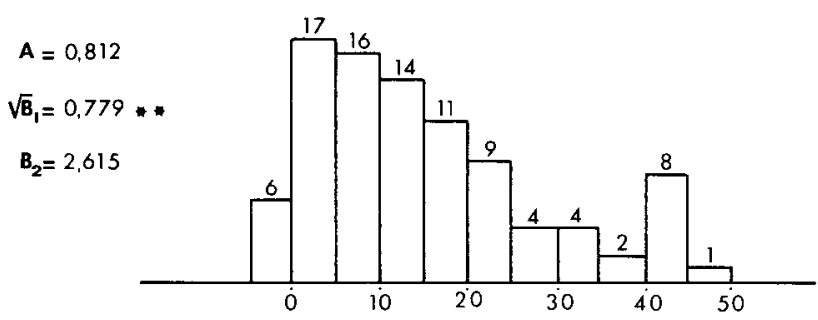

$$
A=0,821
$$

$\sqrt{B_{1}}=0,036$

$\mathrm{B}_{2}=2,140$ *

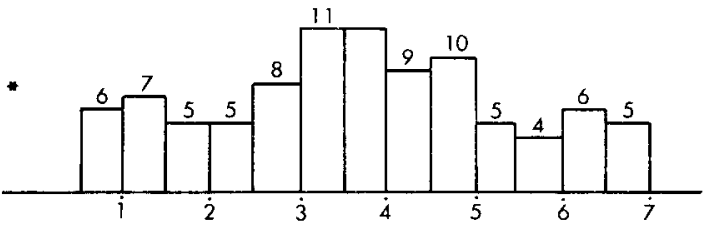

Figure 3

Distribution des nombres de larves par inflorescence principale sur l'ensemble des plantes pollinisées infestées en 1980, avant et après transformation en racine carrée.

Distribution of number of larvae per main shoot, for all pollinated and attacked plants in 1980, before and after square root transformation.

\section{Estimation du nombre utile de plantes par cage}

Si l'on considère que la variance de la production des témoins (en 1980) est une bonne estimation de la variance qu'aurait eue la population en l'absence d'infestation, on peut calculer le nombre d'individus nécessaires dans 2 lots de plantes pour mettre en évidence une différence donnée ( $\delta$ ) par rapport à la moyenne des témoins avec une probabilité donnée, $\beta$, et un risque $\alpha$.

Avec $\beta=0,9$ et $\alpha=0,05$, pour une différence de $1,44 \mathrm{~g}$ ( 5 p. 100), il faudrait 785 plantes environ dans chaque lot, en données brutes. Une différence de 5 p. 100 sur données transformées (représentant ici 15 p. 100 en données brutes) serait mise en évidence dans les mêmes conditions avec 75 plantes. En faisant le calcul inverse, on peut montref que
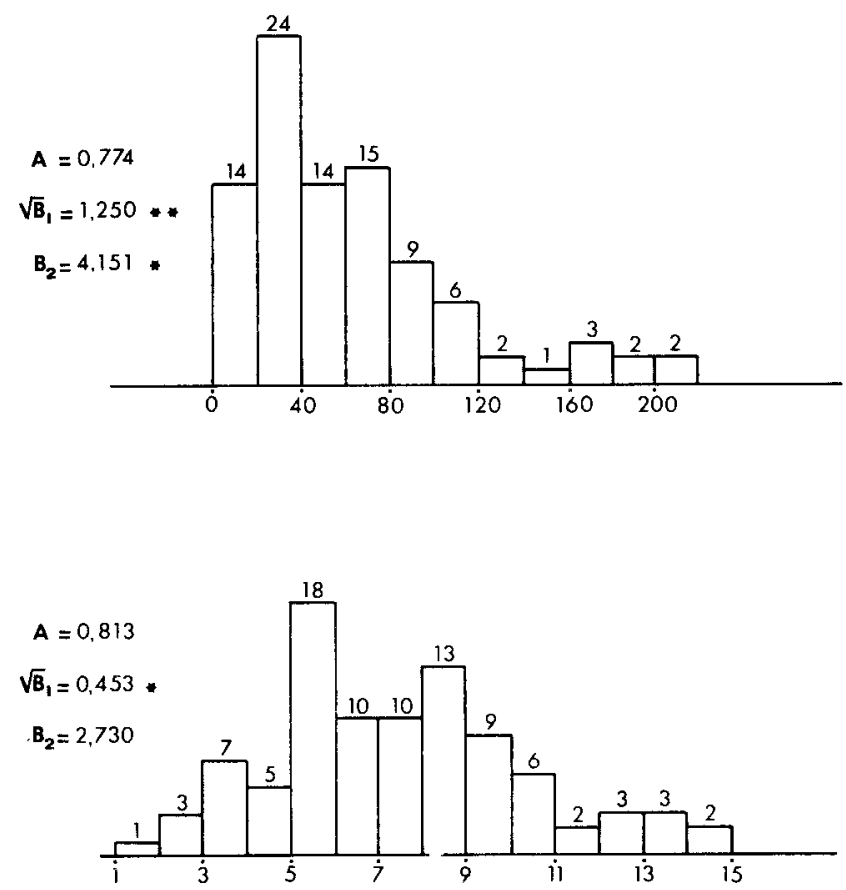

Figure 4

Distribution des nombres de larves par plante sur l'ensemble des plantes pollinisées et infestées en 1980, avant et après transformation en racine carrée.

Distribution of number of larvae per plant, for all pollinated and attacked plants in 1980, before and after square root transformation.

le dispositif de 1980 avec 16 plantes par cage ne pouvait permettre de mettre en évidence dans 9 cas sur 10 que des différences d'environ $10 \mathrm{~g}$ pour une production moyenne de $28,8 \mathrm{~g}$, soit environ 35 p. 100 . Pour 1979 , les estimations sont du même ordre quoiqu'un peu plus faibles.

Il faut donc récolter un très grand nombre de plantes si on veut mettre en évidence les différences de rendement relativement faibles causées par des niveaux d'infestation larvaire susceptibles d'être rencontrés dans les champs. Cependant, les corrélations fortes entre nombre de siliques et production peuvent permettre d'augmenter la précision des tests en utilisant l'analyse de covariance, où la covariable sera le nombre de siliques. Celui-ci déterminant en moyenne 73 p. 100 de la variance du rendement, on doit sensiblement améliorer les résultats. Cette approche n'est valable que dans la mesure où l'on admet que l'infestation n'a pas d'influence notable sur le nombre de siliques.

La solution préconisée pour d'autres études (à venir) consisterait à multiplier les lots (les cages ou les prélèvements groupés au champ) et à travailler sur les moyennes des lots. La moyenne d'une cage sera calculée simplement 
en prenant le rendement total du lot, divisé par le nombre de plantes. Les écarts entre lots seront certainement moins importants qu'entre plantes à l'intérieur d'un lot. Dans tous les cas, il est primordial de définir un système d'échantillonnage des larves caractérisant le niveau d'infestation des plantes, l'observation silique par silique d'un nombre important de plantes étant matériellement impraticable. Cette méthode d'échantillonnage passe par une bonne compréhension de la distribution des larves sur les plantes qui est présentée ci-dessous.

\section{Distribution des larves sur les plantes. Nombre de larves par femelle}

La répartition des larves sur les inflorescences principales d'une cage n'est pas liée au nombre de siliques portées par celles-ci, sauf lorsque la densité d'adultes est importante par rapport au nombre potentiel de lieux de ponte, comme en 1979, c'est-à-dire lorsqu'il y a compétition. Aucune des corrélations entre nombre de siliques et nombre de larves n'est significative en 1980. Il n'est donc pas utile de les présenter ici. Il n'y a pas non plus de corrélations stables et significatives avec aucune des autres variables mesurées (diamètre au collet et nombre d'inflorescences) qui pourraient indiquer un choix de la plante, à ce stade, sur des critères de vigueur perceptibles par le charançon.

En revanche, il y a une relation certaine entre le développement total d'une plante et le nombre de larves qu'elle porte, même pour des niveaux d'infestation très bas (tabl. 5). Le nombre de larves final ne dépend que dans une certaine mesure du nombre d'adultes initial ; il est largement influencé par le développement des plantes. Divers auteurs (RISBEC, 1952 ; BONNEMAISON, 1957 ; DMOCH, 1965 ) ont montré que la durée de la ponte chez $C$. assimilis dépend de la possibilité pour l'insecte de se nourrir d'organes jeunes, boutons et fleurs notamment. Les insectes quittent les parcelles les plus avancées pour aller sur des colzas tardifs dans le cas de semis échelonnés ou sur les colzas de printemps : plus la floraison sera étalée dans le temps, plus la ponte sera abondante. La présence d'inflorescences tertiaires qui prolongent la floraison dans certaines conditions climatiques peut également entraîner une reprise de la ponte.

Le nombre de siliques total et le nombre d'inflorescences, qui donnent une indication sur la durée de floraison, ont été portés dans le tableau 6 qui présente les résultats totaux sur l'effectif complet par cages (16 plantes), mais seules les plantes ayant des inflorescences principales ont été prises en considération pour la dernière colonne.

Le faible nombre de larves par femelle en 1979 peut être expliqué par la date d'infestation qui était tardive (fin de floraison). Pour 1980, l'interprétation est plus complexe. Elle doit prendre en compte non seulement le nombre de siliques total, mais également des différences de mortalité des femelles d'autant plus appréciables que la mort survient tôt et dans de petits effectifs. La comparaison des 2 lots à 1 insecte par plante, avec et sans bourclon, confirme l'influence de la durée de floraison, les plantes non pollinisées fleurissant beaucoup plus longtemps que les autres (LERIN, 1982). Le nombre de larves sur les infiorescences principales, rapporté au nombre de femelles initial est remarquablement constant pour une année donnée, à l'exception, pour 1980, de la cage 1 du traitement à 2 charançons par plante où l'hypothèse d'une mortalité précoce de plusieurs femelles n'est pas à exclure. La variation de 27 p. 100 entre la valeur la plus faible et la plus forte de 1980 pour le lot pollinisé (exception faite de la cage mentionnée précédemment) est en effet négligeable par rapport aux écarts sur les nombres totaux.

Nous avons donc des infestations qui, sur les inflorescences principales, dépendent essentiellement du nombre initial de femelles et, sur les plantes entières, d'une interaction entre développement du végétal et aptitude à pondre des femelles. La résultante de cette interaction étant une intensité de liaison constante entre nombre de siliques et nombre de larves. La comparaison des coefficients de régression établis sur les données transformées montre que ceux-ci ne sont pas significativement différents : les droites sont parallèles et ne diffèrent que par leur ordonnée à l'origine. Les valeurs de celles-ci pour les cages en 1979 et 1980 (pollinisé) sont corrélées $(\mathrm{r}=0,950,6 \mathrm{~d}$.l) avec la densité initiale des adultes en début de traitement. Autrement dit, pour un traitement donné, on aura un rapport constant entre nombre de siliques et nombre de larves. C'est cette relation qui est développée au paragraphe suivant.

\section{E. Détermination simplifiée du niveau d'attaque}

Le niveau d'attaque est défini ici comme le rapport du nombre de larves sur le nombre de siliques. Ce rapport peut

TABLEAU 5

Corrélations entre nombre total de siliques (ST) et nombre total de larves (LT) par plante et par cage après transformation de ST en Log et de LT en $\sqrt{L T+0,5}$

Correlation between total number of pods (ST) and total number of larvae (LT) per plant and per cage after logarithmic transformation of ST and transformation of $L T$ into $\sqrt{L T+0.5}$.

\begin{tabular}{|c|c|c|c|c|c|c|c|}
\hline Année & & Nbre d'insectes/plante & Cage 1 & & ge 2 & $\begin{array}{l}\text { Estimation } \\
\text { moyenne }\end{array}$ & $\begin{array}{l}\text { Coefficient } \\
\text { de régression }\end{array}$ \\
\hline 1979 & & 2,5 & (16) 0,677 & (14) & 0,711 & 0,693 & 3,73 \\
\hline 1980 & $\mathrm{NP}$ & 1 & (13) 0,689 & (15) & 0,798 & 0,753 & 3,45 \\
\hline 1980 & $\mathrm{P}$ & $\begin{array}{l}0,5 \\
1 \\
2\end{array}$ & $\begin{array}{ll}(14) & 0,619 \\
(15) & 0,552 \\
(12) & 0,766\end{array}$ & $\begin{array}{l}(16) \\
(16) \\
(14)\end{array}$ & $\begin{array}{l}0,815 \\
0,556 \\
0,861\end{array}$ & 0,714 & 3,82 \\
\hline
\end{tabular}

\footnotetext{
NP plantes non pollinisées.
}

$P$ plantes pollinisées.

Le nombre d'individus est indiqué entre parenthèses devant le coefficient de corrélation. 
TABLEAU 6

Nombre de larves par femelle et par cage.

Number of larvae per female and per cage.

\begin{tabular}{|c|c|c|c|c|c|c|c|}
\hline Année & & $\begin{array}{l}\text { Nbre d'insectes } \\
\text { par plante }\end{array}$ & Cage & $\begin{array}{l}\text { Nbre larves } \\
\text { par femelle }\end{array}$ & $\begin{array}{l}\text { Nbre siliques } \\
\text { par plante }\end{array}$ & $\begin{array}{l}\text { Nbre d'inflorescences } \\
\text { par plante }\end{array}$ & $\begin{array}{l}\text { Nbre larves sur IP } \\
\text { par femelle }\end{array}$ \\
\hline 1979 & & 2,5 & $\begin{array}{l}1 \\
2\end{array}$ & $\begin{array}{l}56,5 \\
60,9\end{array}$ & $\begin{array}{l}114,6 \\
130,0\end{array}$ & $\begin{array}{l}4,56 \\
4,93\end{array}$ & $\begin{array}{l}17,1 \\
14,5\end{array}$ \\
\hline 1980 & $\mathbf{P}$ & $\begin{array}{l}0,5 \\
1 \\
2\end{array}$ & $\begin{array}{l}1 \\
2 \\
1 \\
2 \\
1 \\
2\end{array}$ & $\begin{array}{r}120,5 \\
193,3 \\
72,6 \\
88,1 \\
92,6 \\
147,9\end{array}$ & $\begin{array}{l}311,8 \\
348,8 \\
302,6 \\
218,2 \\
316,8 \\
321,6\end{array}$ & $\begin{array}{l}9,88 \\
9,88 \\
8,94 \\
8,38 \\
9,63 \\
9,44\end{array}$ & $\begin{array}{l}24,8 \\
33,0 \\
24,6 \\
33,5 \\
18,0 \\
30,5\end{array}$ \\
\hline 1980 & $\mathbf{N P}$ & 1 & $\begin{array}{l}1 \\
2\end{array}$ & $\begin{array}{l}114,0 \\
177,6\end{array}$ & $\begin{array}{l}359,6 \\
320,6\end{array}$ & $\begin{array}{r}9,82 \\
10,38\end{array}$ & $\begin{array}{l}31,1 \\
34,3\end{array}$ \\
\hline
\end{tabular}

$P$ pollinisé

NP non pollinisé.

IP inflorescence principale.

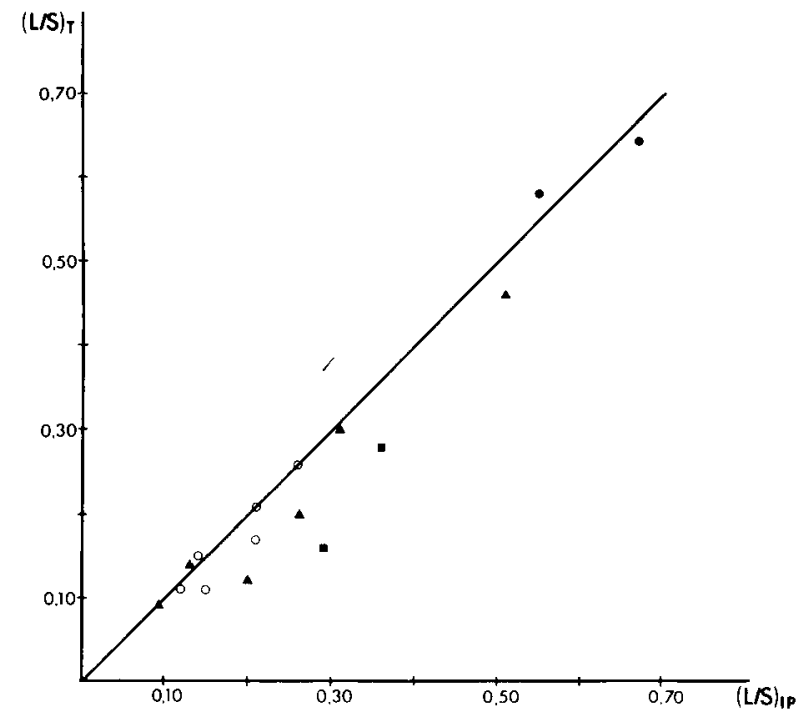

Figure 5

Niveau d'infestation larvaire de la plante entiere, $(L / S) T$, en fonction de celui de l'inflorescence principale, (L/S)I.P, moyenne par cage. Infestation level of the main shoot, (L/S)I.P, compared with infestation level of the whole plant, $(L / S) T$.

- 1979

A 1980 pollinisé (pollinated)

- 1980 non pollinisé (unpollinated)

O 1981 plantes ayant au moins 2 inflorescences secondaires (plant with at least 2 secondary racemes).

être supérieur à 1. En effet, on peut avoir jusqu'à 3 larves dans une silique dans les cas de très fortes infestations. Cependant, pour obtenir cette valeur sur l'ensemble de la plante, il faudrait une densité d'insectes peu susceptible d'être rencontrée dans la nature : en 1979, pour 2,5 adultes par plante, le niveau maximum atteint sur inflorescence principale a été de 1,14 et de 1,0 pour la plante entière la plus attaquée. C'est pourquoi il semble préférable de ne pas exprimer ce rapport en pourcentage d'attaque.

Il n'y a pas de corrélation entre le nombre de larves de l'inflorescence principale et le nombre de larves des inflorescences secondaires, résultat attendu, puisque la ponte sur l'inflorescence principale semble se faire au hasard et que le nombre de larves sur les inflorescences secondaires dépend en partie du développement de la plante.

Il existe une bonne relation entre les moyennes par cage des niveaux d'attaque des inflorescences principales et celles des inflorescences secondaires : $r=0,967$ pour $12 \mathrm{~d} .1$ ( 3 années d'expérimentation, $\mathrm{cf}$ ci-dessous). D'autres regroupements des plantes donnent des résultats similaires. Mais la relation à l'intérieur de chaque cage n'est significative que pour les cages les plus infestées ( 1 cage en 1980, 2 cages en 1979).

Des différences d'attractivité biochimique et des différences de précocité ou de durée de la floraison pourraient expliquer certaines distorsions par rapport aux droites de régression. Mais aucune corrélation n'a pu être établie, ni avec les teneurs en isothiocyanates (butenyl ITC et pentenyl ITC) ni en vinylthiooxazolidone (V.T.O.) des graines récoltées.

En revanche, il existe une assez bonne relation entre le niveau d'attaque de l'inflorescence principale et le niveau d'attaque total pour chaque cage (tabl. 7).

Le fait que le niveau d'attaque de l'inflorescence principale participe au niveau d'attaque global n'est pas gênant puisqu'il s'agit de déterminer ce dernier. Cela revient à évaluer l'attaque sur $31,5 \mathrm{p}$. 100 des siliques en 1979 et 23,8 p. 100 en 1980 , ces chiffres représentant le pourcentage de siliques portées par l'inflorescence principale par rapport au total.

Lorsque les plantes sont regroupées en lots (par exemple la cage), comme dans la figure 5, les variations entre plantes se tamponnent et la corrélation devient bien meilleure, les points se répartissant autour de la bissectrice. Les résultats de la récolte 1981 ont été portés sur le graphique à titre de confirmation: leur bon alignement avec les autres est d'autant plus intéressant que les conditions d'expérience étaient très différentes; les 6 cages ont été posées sur un champ et 100 charançons introduits progressivement dans chacune d'elles. Seules les plantes des échantillons prélevés ayant 2 inflorescences au moins sont prises en compte ; les valeurs sont présentées dans le bas du tableau 7 .

D'autres regroupements ont été faits pour s'assurer de la 
TABLEAU 7

Corrélations entre niveau d'attaque de l'inflorescence principale et niveau d'attaque total, par plante et par cage. Correlation between infestation level on the main shoot and total infestation level per plant and per cage.

\begin{tabular}{|c|c|c|c|c|c|c|c|}
\hline Année & $\begin{array}{l}\text { Nbre d'insectes } \\
\text { par plante }\end{array}$ & Cage & $\begin{array}{l}\text { Nbre de } \\
\text { plantes }\end{array}$ & Corrélation & Signification & $\begin{array}{l}\text { Niveau moyen } \\
\text { sur IP }\end{array}$ & $\begin{array}{l}\text { Niveau moyen } \\
\text { total }\end{array}$ \\
\hline \multirow[t]{2}{*}{1979} & 2,5 & 1 & 13 & 0,789 & $\mathbf{x x}$ & 0,55 & 0,58 \\
\hline & & 2 & 15 & 0,822 & $\mathrm{xx}$ & 0,67 & 0,65 \\
\hline \multirow[t]{6}{*}{1980} & 0,5 & 1 & 14 & 0,595 & $\mathbf{x}$ & 0,13 & 0,14 \\
\hline & & 2 & 16 & 0,692 & $\mathrm{xx}$ & 0,09 & 0,09 \\
\hline & 1 & 1 & 15 & 0,543 & $\mathbf{x}$ & 0,20 & 0,12 \\
\hline & & 2 & 16 & 0,736 & $\mathbf{x x}$ & 0,26 & 0,20 \\
\hline & 2 & 1 & 12 & 0,778 & $\mathbf{x x}$ & 0,31 & 0,30 \\
\hline & & 2 & 14 & 0,798 & $\mathrm{xx}$ & 0,51 & 0,46 \\
\hline \multirow[t]{2}{*}{1980} & 1 & 1 & 13 & 0,436 & & 0,36 & 0,28 \\
\hline & & 2 & 15 & 0,565 & $\mathbf{x}$ & 0,29 & 0,16 \\
\hline \multirow[t]{6}{*}{$1981 \quad \mathrm{P}$} & $0,6\left(^{1}\right)$ & 1 & 17 & 0,749 & $\mathbf{x x}$ & 0,21 & 0,17 \\
\hline & & 2 & 19 & 0,881 & $\mathbf{x x}$ & 0,14 & 0,15 \\
\hline & & 3 & 18 & 0,781 & $\mathbf{x x}$ & 0,15 & 0,11 \\
\hline & & 4 & 13 & 0,815 & $\mathbf{x x}$ & 0,26 & 0,26 \\
\hline & & 5 & 11 & 0,882 & $x x$ & 0,21 & 0,21 \\
\hline & & 6 & 12 & 0,612 & $\mathrm{xx}$ & 0,12 & 0,11 \\
\hline
\end{tabular}

IP inflorescence principale.

$P$ pollinisé.

NP non pollinisé.

Pour 1981 ne sont prises en compte que les plantes ayant au moins 2 inflorescences secondaires.

Seuil de signification : $5 \% \mathrm{x}, 1 \% \mathrm{xx}$.

(1) Sex ratio inconnu.

validité de la corrélation entre niveau d'attaque de l'inflorescence principale et niveau d'attaque global:

1) moyennes des plantes (7 à 8 ) occupant la même position dans les différentes cages quel que soit le traitement en $1980: n=16, r=0,982$

2) moyennes établies sur des groupes de 7 à 8 plantes formés dans chaque cage :

- en 1980 (témoins sans charançon exclus) $n=12$, $\mathrm{r}=0,982$

- en $1979 \mathrm{n}=4, \mathrm{r}=0,992$

Enfin, il existe une très bonne relation entre le nombre d'adultes initial et le niveau d'attaque sur inflorescence principale (tabl. 8).

Il faut noter que l'absence de pollinisation introduit une erreur dans l'estimation du niveau d'attaque global (tabl. 7 et fig. 5) : la floraison des inflorescences principales et donc la production de siliques jeunes se sont étendues pratiquement jusqu'à la récolte. Comme les insectes montrent une préférence marquée pour ces inflorescences pendant leur floraison (DMOCH, 1965 ; GouLD, 1975), la ponte s'est poursuivie plus longtemps à ce niveau et le niveau d'attaque est plus élevé que sur le reste de la plante.

Cela indique que ce mode d'échantillonnage du niveau d'attaque n'est valable que pour des comparaisons à l'intérieur d'un lot de plantes homogènes (cages, parcelle ou champ), de la même variété et pour une population d'adultes arrivant précocément, c'est-à-dire avant la floraison ou à son début. Il faudra confirmer cette relation en conditions de plein champ où les insectes ont une plus grande liberté de choix et peuvent se reporter sur des parties plus tardives de la parcelle. Cependant, les résultats présentés par Gould (1975) sur 3 années en plein champ confir- ment la bonne estimation du niveau d'attaque total par celui de l'inflorescence principale. La méthode d'échantillonnage utilisée par cet auteur est encore allégée puisqu'il ne prend que 10 siliques par type d'inflorescence pour analyser les plantes. Mais WINFIELD (1961) sur des cultures de printemps de moutarde brune (Brassica juncea Coss.) constate que l'estimation du niveau d'attaque des siliques par un échantillonnage sur les inflorescences principales surévalue systématiquement l'attaque de la plante entière. Nos observations sur colza de printemps vont dans le même sens. La méthode ne s'appliquerait donc qu'à des populations de charançons jeunes dont le potentiel de reproduction est intact, ce qui n'est pas le cas pour les cultures de printemps.

Il semble néanmoins que l'analyse de l'inflorescence principale soit suffisante pour déterminer avec précision le niveau d'attaque des plantes pour le colza d'hiver. Dans le cas de prélèvements nombreux, par exemple d'échantillons

\section{TABLEAU 8}

Relation entre le nombre initial d'adultes et le niveru d'attaque de l'inflorescence principale par traitement.

Relation between initial number of adults and infestation level on the main shoot, for each treatment.

\begin{tabular}{cccc}
\hline \hline Année & $\begin{array}{c}\text { Nbre d'adultes } \\
\text { par plante }\end{array}$ & $\begin{array}{c}\text { Niveau d'attaque } \\
\text { sur IP }\end{array}$ & $\begin{array}{c}\text { Intervalle } \\
\text { de confiance }\end{array}$ \\
\hline 1980 & 0,5 & 0,115 & $\pm 0,031$ \\
& 1 & 0,233 & $\pm 0,071$ \\
& 2 & 0,429 & $\pm 0,068$ \\
\hline 1979 & 2,5 & 0,589 & $\pm 0,075$ \\
\hline
\end{tabular}


de $1 \mathrm{~m}$ dans la ligne de semis (entre 15 et 30 plantes), cela apporterait une diminution considérable du temps de dépouillement et devrait permettre d'établir une relation entre rendement et niveau d'attaque.

\section{CONCLUSION}

Pour des expériences précises visant à déterminer l'impact des larves de charançon sur le rendement du colza, la transformation logarithmique du nombre de siliques, du nombre de graines et du rendement de la plante entière apparaît nécessaire, ainsi que la transformation en racine carrée du nombre de larves. En revanche, pour les inflorescences principales, cette transformation n'est pas utile.

Une méthode pratique pour estimer les pertes de récolte dues au charançon des siliques ne peut passer par un dénombrement exhaustif des diverses composantes du rehdement d'un ensemble de plantes entières, principalement à cause de la longueur du travail de dépouillement. L'acquisition de données plus sommaires mais portant sur un plus grand nombre de lots de plantes est possible si l'on n'examine que les inflorescences principales.

Cet échantillonnage permet :

1) le comptage des plantes d'un lot,

2) la détermination du niveau d'attaque qui pourra être relié au rendement,

3) une estimation du nombre de graines par silique caractérisant le niveau de production et de pollinisation sans risque d'égrenage en cas de prélèvement tardif, puisque les inflorescences principales ne s'entremêlent pas. Il faut, dans ce cas, inclure dans les comptages les graines détruites par les charançons.

L'estimation par cette méthode du nombre de graines par silique est légèrement biaisée par le fait que l'inflorescence principale est toujours plus productive que les autres (CLARKE, 1979: LERIN, 1982), mais elle peut permettre de prévoir un niveau de risque. En effet, la larve de charançon doit consommer de 3,5 à 5 graines (selon les auteurs) pour son complet développement. Cette estimation n'est pas susceptible de varier énormément. En revanche, les cultures de colza ont des nombres de graines par silique variant de plus du double suivant les conditions climatiques et de fertilisation (entre 10 et 25 graines par silique). Pour un même taux de siliques attaquées, on voit que l'impact du charançon sur la production sera très différent suivant le nombre de graines par silique, si l'on néglige une hypothétique compensation des dégâts par les plantes.

Reçu le 11 mars 1982 Accepté le 27 juillet 1982

\section{REMERCIEMENTS}

Je tiens à remercier M. RENARD et $M$. DESChAMPS de la Station d'Amélioration des Plantes de Rennes (I.N.R.A.) pour le travail d'analyse des glucosinolates des graines.

\section{RÉFÉRENCES BIBLIOGRAPHIOUES}

Bonnemaison L., 1957. Le charançon des siliques (Ceuthorrhynchus assimilis Payk.). Biologie et méthode de lutte. Ann. Epiphyt., 387543.

Buson M., 1979. Hétérosis et paramètres génétiques de quelques caractères agronomiques chez le colza oléagineux (Brassica napus L.). Thèse de doctorat de $3^{\mathrm{e}}$ cycle. Université Pierre et Marie Curie, Paris VI, 78 p. + annexes.

Clarke J. M., 1979. Intraplant variation in number of seeds per pod and seed weight in Brassica napus cv « Tower». Can. J. Plant Sci., 59, 959-962.

Clarke J. M., Simpson G. M., 1978. Influence of irrigation and seeding rates on yield and yield components of Brassica napus cv «Tower ». Can. J. Plant Sci., 58, 731-737.

Dagnelie P., 1975. Théorie êt méthodes statistiques. Les Presses Agronomiques de Gembloux.

Dembinski F., 1970. Influence des périodes d'insuffisance d'eau dans le sol en automne et au printemps sur le développement et le rendement du colza d'hiver. $C$. $R$. Journées Internationales sur le colza, 104-111.

Dmoch J., 1965. The dynamics of a population of the cabbage seed pod weevil (Ceuthorrhynchus assimilis Payk.) and the development of winter rape. Part I \& II. Ekol. pol., sér. A, 13, 249-287, 463-489.

Elliott J. M., 1971. Some methods for the statistical analysis of samples of benthic invertebrates. Freshwater biological association. Scientific Publication $\mathrm{n}^{\circ} 25,144 \mathrm{pp}$.
Gould H. J., 1975. Surveys of pest incidence on oil seed rape in South Central England. Ann. appl. Biol., 79, 19-26.

Lacotte J. P., 1973. Mise au point de la lutte raisonnée contre les insectes ravageurs du colza. Rapport d'activité CETIOM

Lerin J., 1982. Effets de la pollinisation entomophile sur le colza dans une expérience en cage. Agronomie, 2 (3), 249-256.

Pielou E. C., 1969. An introduction to mathematical ecology. Wiley Interscience, $286 \mathrm{pp}$

Risbec J., 1952. Contribution à l'étude de Ceuthorrhynchus assimilis Payk, charançon des siliques du colza. Rev. Pathol. veg. Entomol. agric., 31, 137-174.

Rollier M., 1974. Influence des facteurs climatiques sur le rendement du colza d'hiver. Inf. tech. CETIOM, 37, 9-12.

Southwood T. R. E., 1966. Ecological methods. Methuen \& Co Ltd, 8-39 (391 pp.).

Williams I. H., Free J. B., 1979. Compensation of oil seed rape (Brassica napus L.) plants after damage to their buds and pods. $J$. agric. Sci., Camb., 92, 53-59.

Winfield A. L., 1961. Field observations of the control of blossom beetles (Meligethes aeneus F.) and cabbage-seed weevil (Ceuthorrhynchus assimilis Payk) on mustard-seed crops of East Anglia. Ann. appl. Biol., 49, 539-555. 